\title{
Chronic respiratory morbidity after prolonged and premature rupture of the membranes
}

\author{
P J Thompson, Anne Greenough, K Nicolaides
}

\begin{abstract}
Twenty one surviving infants of pregnancies complicated by rupture of the membranes during the second trimester that lasted at least one week have been followed up for a median of 15 months. Five infants (24\%) had recurrent respiratory problems (episodes of wheezing and coughing occurring at least once a week) which related significantly to the use of neonatal ventilation and to very preterm delivery. Five of the 18 infants who were born preterm and with birth weights of $<2500 \mathrm{~g}$ had recurrent respiratory symptoms (28\%). This compares favourably with an incidence of symptoms of $67 \%$ among surviving low birthweight infants born at this hospital after pregnancies not complicated by premature rupture of the membranes. Neither recurrent respiratory symptoms nor admission to hospital for chest related disorders were associated with the timing of onset or duration of rupture of the membranes. We conclude that, among survivors of premature rupture of the membranes, chronic respiratory morbidity would best be prevented by avoiding very preterm delivery, regardless of the duration of the rupture.
\end{abstract}

Premature rupture of the membranes (rupture of the membranes before 37 completed weeks' gestation) has been estimated to occur in $2-3 \%$ of pregnancies. ${ }^{1}$ This may result in oligohydramnios, which is associated with pulmonary hypoplasia. ${ }^{2}{ }^{3}$ Pulmonary hypoplasia is often fatal but there are reports of infants surviving after premature rupture of the membranes, even after rupture as early as 15 weeks and oligohydramnios that persisted for as long as 20 weeks. ${ }^{2-4}$ Many of these infants, however, had respiratory problems in the neonatal period but, as most were born prematurely, the diagnosis seemed likely to be respiratory distress syndrome. These data do not, however, exclude minor degrees of abnormal lung growth. Long term follow up data on survivors of such pregnancies are not available, so it is not possible to be certain that such children are not at increased risk of respiratory infections or recurrent respiratory problems.

The aim of this prospective study was to follow up infants born to mothers who had prolonged premature rupture of the membranes to see if the children had signs of chronic respiratory disease.

King's College Hospital, London SE5 9PJ Department of Child Health P J Thompson Anne Greenough

Harris Birthright Centre K Nicolaides

Correspondence to: Dr Greenough.

Accepted 3 April 1990 intrauterine death, and 22 neonatal deaths (20 of pulmonary hypoplasia and two of sepsis). Pulmonary hypoplasia was diagnosed at necropsy using the methods we have described previously ${ }^{4}$; in brief, by a reduction in lung:body weight ratio of less than 0.012 and a reduced radial alveolar count (less than $4 \cdot 1$ ).

Twenty six children were discharged home, 21 of whom have been followed up for a median of 15 months (range 6-22). In five cases the children were lost to follow up, but none of these children had been ventilated in the neonatal period or had been admitted to the neonatal intensive care unit. In the 21 infants that we followed up prospectively, rupture of the membranes occurred at a median gestational age of 24 weeks (range 15-32) and delivery at a median gestational age of 32 weeks (range 25-41). The median duration of rupture of the membranes was five weeks (range 1-21) (figure). The infants had a median birth weight of $1670 \mathrm{~g}$ (range 756-3700). Seven infants were ventilated in the neonatal period and these children were born at an earlier gestational age (median 27 weeks, range 25-32) than the remaining 14 infants (median 32.5 weeks, range 30 41 ), $(p<0.005)$, but the gestation at the time of rupture was similar in the two groups (median 24 weeks, range 15-28, and median 27 weeks, range 17-32, respectively). The median duration of rupture was shorter in the ventilated infants, being three weeks (range 1-15) compared with six weeks (range 1-21) in the nonventilated infants $(p<0.05)$.

This study was approved by the King's College Hospital ethics committee.

All children were reviewed regularly in outpatient clinics at six monthly intervals, or more

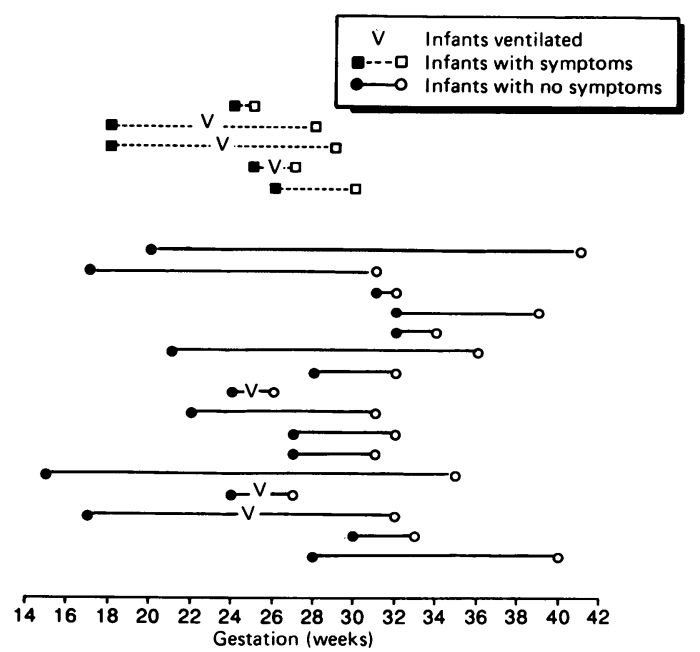

Gestational age at onset of rupture of the membranes (closed symbols) and delivery (open symbols) for 21 surviving infants. The data points depicting onset and delivery are linked for each case to show the duration of rupture. 
often if clinically indicated. At each visit the infants were examined and their height and weight recorded. The parents were asked about the presence and frequency of respiratory problems (coughing and wheezing), if their children had received treatment for such problems, or if they had required admission to hospital. The infants were defined as having symptoms if they had recurrent respiratory symptoms, defined as cough or wheeze, or both, at least once a week, or episodes of coughing or wheezing at least monthly since the time of discharge home and thus had symptoms at each outpatient attendance.

To find out if chronic respiratory morbidity was increased among infants born after pregnancies complicated by premature rupture of the membranes, their results were compared with those of very low birthweight (VLBW) infants born at this hospital during a six month period within the present study period. Results were available from prospective follow up of 44 of 48 consecutive VLBW infants, whose median gestational age was 27 weeks (range 24-34). Infants were described as having symptoms if they fulfilled the same definition as that used for the study group. Results were also available from a retrospective review of 101 preterm low birthweight (LBW) infants whose median gestational age was 31 weeks (range 24-37).

Differences between infants with and without symptoms were assessed for significance with Fisher's exact test and the Wilcoxon rank sum test. Differences in the incidence of symptoms between the study group and the prospective and retrospective follow up of VLBW and LBW infants, respectively, were assessed with Fisher's exact test or the $\chi^{2}$ test with Yates's correction, as appropriate.

\section{Results}

Only one child died during the follow up period: this was at the age of 6 months, and the history, clinical, and necropsy findings were compatible with sudden infant death syndrome. This infant had had no respiratory symptoms or hospital admissions since discharge from the neonatal unit.

Five children had symptoms throughout their period of follow up, having respiratory symptoms at least once a week; three had episodes of coughing, one of wheezing, and one of both coughing and wheezing; the latter infant required regular outpatient supervision and chest physiotherapy. Four of the five children with symptoms had been ventilated during the neonatal period, compared with three of the 16 children who were symptom free $(p<0 \cdot 01)$. The median gestational age at delivery was significantly earlier in the infants with symptoms, being 28 weeks (range 25-30) compared with 32 weeks (range $26-41)$ in the symptom free group $(p<0.05)$; there was, however, no significant difference in the gestational age at the time of rupture (24 weeks (range 18-26) and 27 weeks (range 15-32), respectively), or the duration of rupture (4 weeks (range 1-11) and 6 weeks, range (1-21), respectively).

Only three children required admission to hospital for chest related problems (all infections) during the study period; two of these had been ventilated as neonates and all three had recurrent respiratory symptoms. None of the symptom free children had been readmitted. There was no significant relationship between hospital admission and the time of onset or duration of premature rupture of the membranes or the gestational age at delivery.

There was a lower incidence $(24 \%)$ of infants with symptoms in our study population than in the VLBW and LBW control group. Twenty three of the $44(52 \%)$ VLBW infants from the prospective follow up had symptoms at follow up $(p=0.054)$, as did 68 of the $101(67 \%) \mathrm{LBW}$ infants from the retrospective follow up $(p<0.001)$.

\section{Discussion}

Previous studies that have followed up survivors of premature rupture of the membranes have suggested that such infants may be at increased risk of chronic lung disease. ${ }^{5-8}$ In one series, seven of 17 infants survived with chronic lung disease; 18 infants, however, had been lost to follow up. Unfortunately no definition of chronic lung disease was given, thus it is difficult to make a comparison with the present series. None of our survivors had bronchopulmonary dysplasia as defined by Northway et al, ${ }^{9}$ but three of the 21 survivors (14\%) had chronic lung disease, which was defined as oxygen dependence at 28 days of age together with an abnormal chest radiograph. ${ }^{10}$ The incidence of chronic lung disease among VLBW infants at this hospital is $15 \%$. $^{11}$

Unfortunately none of the previous four studies stated whether respiratory symptoms occurred during follow up of survivors of premature rupture of the membranes. ${ }^{5-8}$ The present series shows, for the first time, that roughly $24 \%$ of such infants have recurrent respiratory symptoms of coughing and wheezing during the first year of life. The infants included in our series were born at varying gestational ages and some even at full term. Eighteen of the 21 patients, however, were born preterm and had birth weights $<2500 \mathrm{~g}$, and although all five infants with symptoms were in this group, it still meant that only $28 \%$ of these preterm infants born following premature rupture of the membranes had recurrent respiratory symptoms. Interestingly this incidence of recurrent respiratory symptoms compares favourably with that among VLBW (52\%) and LBW (67\%) survivors at this hospital. Our data suggest therefore that there may be a reduction in respiratory morbidity in LBW infants born following pregnancies complicated by premature rupture of the membranes compared with such infants from pregnancies without this complication. A further study is under way to determine if other variables not investigated in this study might explain the difference in incidence of symptoms between the groups.

Five of the 26 survivors were lost to follow up and this may have influenced our results. One infant had emigrated and the other four had moved without leaving a change of address. None of the five infants had required admission to the neonatal intensive care unit and all had a relatively late onset of rupture of the membranes compared with the remainder of the group. Thus, regardless of the aetiology of recurrent respiratory symptoms, it seems likely that we had examined the infants with the greatest likelihood of chronic respiratory morbidity. The incidence of this problem following prolonged premature rupture of the membranes thus might be even lower.

Recurrent respiratory morbidity among these infants could be the result of abnormal lung growth. Pulmonary hypoplasia is a common patho- 
logical finding following prolonged premature rupture of the membranes, ${ }^{12}$ and the criteria for making this diagnosis at necropsy are well established. ${ }^{13}$ Pulmonary hypoplasia is a difficult diagnosis to make among survivors, particularly as their clinical course may be complicated by preterm delivery and respiratory distress syndrome. Possibly as a consequence, there are no reports of long term follow up of patients with pulmonary hypoplasia other than those cases that are secondary to congenital diaphragmatic hernia. It is therefore impossible to say whether our infants had the characteristic symptoms of this condition in the first year of life. We ${ }^{4}$ and others, ${ }^{2}$ have shown that fatal pulmonary hypoplasia is significantly related to early onset of rupture of the membranes, particularly if it occurs before 26 weeks' gestation. In the present series, rupture occurred significantly earlier in infants with fatal pulmonary hypoplasia than in those who survived and were free of symptoms $(p<0.01)$. Infants with symptoms tended to have later onset of rupture compared with those with fatal pulmonary hypoplasia, but this difference was not significant. In some infants with symptoms, rupture had occurred before 26 weeks' gestation, and this may have impaired lung growth.

The other aetiology of the recurrent symptoms relates to preterm delivery and neonatal respiratory distress. Recurrent respiratory symptoms, ${ }^{14}$ abnormalities of lung function, ${ }^{15}$ an increased risk of infection, ${ }^{16}$ and the hospital readmission rate, ${ }^{17}$ are common among VLBW survivors, particularly those ventilated in the neonatal period and surviving with chronic lung disease. In this study the infants with symptoms were born at a significantly earlier gestational age than those without, and a greater proportion were ventilated in the neonatal period. Only three infants in our series survived with evidence of chronic lung disease, and all were symptomatic at follow up. ${ }^{10}$

Although none of the infants with symptoms were receiving maintenance treatment for their recurrent wheezing and coughing, prescribing habits may have been influenced by their relative youth, ${ }^{18}$ so their lack of treatment is not necessarily a reflection of mild disease. The only three children readmitted to hospital were all among our infants with symptoms, suggesting that their respiratory morbidity was appreciable.

Our results suggest that this respiratory morbidity might be prevented by the avoidance of preterm delivery and neonatal ventilation. Expectant management of pregnancies complicated by premature rupture of the membranes can be accomplished without risk to either mother or fetus, ${ }^{4}$ so elective preterm delivery may be avoided. Unfortunately, however, spontaneous preterm delivery occurs commonly in pregnancies complicated by premature rupture of the membranes. ${ }^{4}$ Prevention of respiratory distress (and hence neonatal ventilation) might be a more successful approach to reducing respiratory morbidity. Randomised trials of giving corticosteroids to the mothers have had variable but generally disappointing results in premature rupture of the membranes. ${ }^{19}$ As yet postnatal treatment with surfactant has not been given to enough infants born after premature rupture of the membranes to allow useful comment to be made about effect. ${ }^{20}$

We conclude that chronic respiratory morbidity does occur after prolonged premature rupture of the membranes. Our results suggest that this is particularly related to very preterm delivery, but do not exclude minor degrees of abnormal lung growth occurring in the survivors.

Dr Peter Thompson, research fellow, was supported by Action Research for the Crippled Child.

1 Drife JO. Preterm rupture of the membranes. Br Med 7 1982; 285:583-4.

2 Perlman $M$, Williams J, Hirsch $M$. Neonatal pulmonary hypoplasia after prolonged leakage of amniotic fluid. Arch hypoplasia after prolonged

3 Thomas IT, Smith DW. Oligohydramnios, cause of the nonrenal features of Potter's syndrome including pulmonary hypoplasia. I Pediatr 1974;84:811-4.

4 Blott M, Greenough A. Neonatal outcome after prolonged rupture of the membranes starting in the second trimester. Arch Dis Child 1988;63:1146-50.

5 Taylor JO, Garite JJ. Premature rupture of membranes before fetal viability. Obstet Gynecol 1984;64:615-20.

6 Beydoun MD, Yasin SY. Premature rupture of the membranes before 28 weeks: conservative management. $A m \mathcal{F}$ Obstet Gynecol 1986;155:471-9.

7 Moretti M, Sibai BM. Maternal and perinatal outcome of expectant management of premature rupture of membranes in the mid-trimester. Am f Obstet Gynecol 1988;159:390-6.

8 Bengston JM, Van Marter LJ, Barss VA, et al. Pregnancy outcome after premature rupture of the membranes at or

before 26 weeks gestation. Obstet Gynecol 1989;73:921-6.

Northway WH, Rosan RC, Porter DY. Pulmonary disease following respiratory therapy of hyalin

10 Bancalari E, Abdenour GE, Feller R, Gannon J. Bronchopulmonary dysplasia. Clinical presentation. I Pediatr 1979; 95:819-24.

11 Greenough A. Chronic lung disease. In: Roberton NRC, ed. Textbook of neonatology. Edinburgh: Churchill Livingstone. (In press.)

12 Wigglesworth JS, Desai R. Is respiratory function a major determinant of perinatal survival? Lancet 1982;i:264-7.

13 Wigglesworth JS, Desai R, Guerrini PF, et al. Lung hypoplasia: biochemical and structural variations and their possible significance. Arch Dis Child 1981;56:606-15.

14 Bowman E, Yu VYK. Continuing morbidity in extremely low birthweight infants. Early Hum Dev 1989;18:165-74.

15 Smyth JA, Tabachnik E, Duncan WJ, Reilly BJ, Levison H Pulmonary function and bronchial hyperactivity in long term survivors of bronchopulmonary dysplasia. Pediatrics 1981;68:336-40.

16 Markestad T, Fitzhardinge PM. Growth and development in children recovering from bronchopulmonary dysplasia. f Pediatr 1981;98:597-602.

17 Ford GW, Rickards AL, Kitchen WH, Lissenden JV, Keith CG, Ryan MM. Handicaps and health problems in two year old children of birth weight 500 to $1500 \mathrm{~g}$. Aust Paediatr $\mathcal{F}$ 1985;21:15-22.

18 Lenney $W$, Milner AD. At what age do bronchodilators work? Arch Dis Child 1978;53:532-5.

19 Ohlsson A. Treatments of preterm premature rupture of the membranes: a meta analysis. Am J Obstet Gynecol 1989 160:890-906.

20 Morley CJ, Greenough A, Miller NJ, et al. Randomised tria of artificial surfactant (ALEC) given at birth to babies from 23 to 34 weeks of gestation. Early Hum Dev 1988;17:41-54.
Having been asked by several mothers about the absence of tears in their babies aged 2 or 3 months or more, having excluded Riley's syndrome, and knowing the frequency with which the eyes of some babies water because the tear duct has not completely opened, I consulted the largest popular paediatric textbook in our hospital library. The only reference to 'Tears' in the index was 'Tear Gas'.

A RETIRED DISTINGUISHED PAEDIATRICIAN-EDS. 